\title{
Defining and Refining the Role for Surgery and Intraperitoneal Chemotherapy in the Treatment of Peritoneal Surface Malignancies
}

\author{
M. Haroon Choudry, MD ${ }^{1}$, David L. Bartlett, $\mathrm{MD}^{1}$, H. Richard Alexander, MD ${ }^{2}$, and Kiran K. Turaga, $\mathrm{MD}^{3}$ \\ ${ }^{1}$ Surgical Oncology, University of Pittsburgh Medical Center, Pittsburgh, PA; ${ }^{2}$ Department of Surgery, Rutgers Cancer \\ Institute of New Jersey, New Brunswick, NJ; ${ }^{3}$ Department of Surgery, University of Chicago, Chicago, IL
}

The 14th Annual International Symposium on Regional Cancer Therapies (2019) took place in Phoenix Arizona, under the auspices of the Society of Surgical Oncology. Once again, this meeting was a tremendous success and provided another opportunity for education, thoughtful discourse, scientific collaboration, and innovation. In this educational series, we highlight some of the recent preclinical and clinical research focused on regional therapies for metastatic cancers.

Khalili et al. ${ }^{1}$ present some elegant preclinical research focused on overcoming the steric hinderance of extracellular mucus that impairs drug delivery to mucinous tumors, such as pseudomyxoma peritonei (PMP). They demonstrate homogenous distribution of modified nanoparticles through PMP tumor explants ex vivo and in subcutaneous tumor nodules of mucin-secreting colon cancer cells in vivo. These modified nanoparticles may represent "an effective mucin penetrating drug delivery system." Another important contribution is a systematic review of the literature by Stein et al., ${ }^{2}$ which summarizes the molecular and genetic markers that have been characterized in low- and high-grade appendiceal mucinous tumors. A more comprehensive molecular understanding of these uncommon tumors is vital to improve subtype

\footnotetext{
(C) Society of Surgical Oncology 2019
}

First Received: 3 October 2019;

Published Online: 23 October 2019

K. K. Turaga, MD

e-mail: kturaga@uchicago.edu classification, provide clinically meaningful prognostic and predictive markers, and identify potential therapeutic targets.

Baratti et al. $^{3}$ provide data from a prospective study comparing cytoreductive surgery (CRS) with or without hyperthermic intraperitoneal chemoperfusion (HIPEC) (60min of mitomycin \pm cisplatin), as a component of multimodality therapy, in patients with colorectal peritoneal metastases. This is a timely study since the recently completed PRODIGE-7 trial failed to show any benefit for oxaliplatin-based HIPEC (for $30 \mathrm{~min}$ ) in such patients. As with the PRODIGE-7 study, the current study fails to show survival benefit with the addition of HIPEC but reinforces the benefits of CRS in well-selected patients. In a similar vein, Enomoto et al. ${ }^{4}$ assess the role of adjuvant HIPEC in select patients thought to be at high risk for subsequent peritoneal metastases following complete resection of the primary tumor. The role for prophylactic/proactive CRSHIPEC in patients at high risk for peritoneal metastases continues to be debated and studied. By comparing their multi-institutional results with historical data and preliminary data from ongoing randomized trials (e.g., PROPHYLOCHIP, COLOPEC), they fail to observe significant benefits from adjuvant HIPEC over observation alone. These data highlight the need for continued basic and clinical research to identify and optimize novel intraperitoneal therapies that may augment surgical resection.

The impact of aggressive surgical interventions like CRS-HIPEC on postoperative quality of life (QOL) remains an important area for research and intervention. Ali et al. ${ }^{5}$ demonstrate continued improvements in QOL parameters after the first 3 months following surgery and that these improvements are significant compared with the 
preoperative status of such patients. Moreover, Bayat et al. ${ }^{6}$ report that bowel-related QOL parameters are not adequately assessed by global QOL questionnaires. They propose additional screening for bowel-related QOL parameters, especially following low anterior resections, with and without ostomies, to improved targeted interventions in such patients.

Defining optimal surveillance strategies following CRSHIPEC is vital to provide cost-effective care without compromising oncologic outcomes. Gamboa et al. ${ }^{7}$ utilize prospectively collected data from the US HIPEC collaborative to provide guidelines for optimal surveillance frequency after CRS-HIPEC for appendiceal or colorectal peritoneal metastases. They show that low-frequency surveillance (every 6-12 months) does not negatively impact survival and is more cost-effective compared with more frequent surveillance. Similarly, the study by Solomon et al. $^{8}$ finds that most recurrences following CRSHIPEC for low-grade appendiceal mucinous neoplasms (LAMN)/PMP occur within the first 3 years, supporting more frequent surveillance in the first few years in such patients.

Zaidi et al. ${ }^{9}$ utilize prospectively collected data from the U.S. HIPEC collaborative to determine risk factors associated with incomplete cytoreduction in patients with appendiceal/colorectal peritoneal metastases and peritoneal mesothelioma. They assigned clinical risk scores to identify low- and high-risk groups for incomplete cytoreduction. This may prove to be a useful tool to "improve patient selection of CRS-HIPEC and circumvent the major morbidity associated with the procedure." Similarly, Sabesan et al. ${ }^{10}$ validate two existing radiographic scoring systems to predict incomplete CRS in patients with peritoneal metastases from low-grade mucinous adenocarcinoma. Such studies will help decrease the risk and cost associated with futile surgical interventions.

The role for CRS-HIPEC in rare cancers remains hard to define. Stiles et al. ${ }^{11}$ retrospectively review their institutional data of multimodality therapy, including perioperative systemic chemotherapy, CRS-HIPEC, and whole-body radiation therapy for desmoplastic small round cell tumors, a rare and highly aggressive mesenchymal tumor. While they demonstrate promising survival data, this aggressive treatment strategy was associated with significant short- and long-term morbidity. Zambrano-Vera et al. ${ }^{12}$ retrospectively review their institutional data of CRS-HIPEC for another goblet cell carcinomas, another rare tumor with high propensity of peritoneal metastases.

The Regional Cancer Therapies meeting emphasizes the management of liver and melanoma metastases in addition to peritoneal metastases. In this series, Narayan et al. ${ }^{13}$ demonstrate higher disease recurrence following complete resection of colorectal metastases in patients with severe inflammatory hepatic parenchymal disease (nonalcoholic fatty liver disease). They stress the importance of modulating patient-specific risk factors to optimize oncologic outcomes. The safety and feasibility of minimally invasive robotic pelvic lymphadenectomy for metastatic melanoma is demonstrated by Miura et al. ${ }^{14}$

In summary, this educational series once again highlights ongoing research to define and refine the role for regional cancer therapies for patients with metastatic cancers. The role for CRS-HIPEC continues to evolve and basic, translational, and clinical research efforts are essential to optimize care of the complex and challenging patients suffering from such devastating cancers. This annual meeting plays an important role for the community of cancer surgeons, presenting the latest research on the surgical management of metastatic cancers and educating surgeons on standards of practice. As such, the meeting has been renamed the SSO Advanced Cancer Therapies meeting, emphasizing the surgical management of all metastatic cancers, but it will continue to emphasize peritoneal, liver, and melanoma metastases.

DISCLOSURES The authors report no conflicts of interest.

\section{REFERENCES}

1. Khalili M, Zhou H, Thadi A, et al. Slippery nanoparticles as a diffusion platform for mucin producing gastrointestinal tumors. Ann Surg Oncol. 2019. https://doi.org/10.1245/s10434-019-0749 3-7.

2. Stein A, Strong E, Gamblin TC, et al. Molecular and genetic markers in appendiceal mucinous tumors: a systematic review. Ann Surg Oncol. 2019. https://doi.org/10.1245/s10434-019-0787 9-7.

3. Baratti D, Kusamura S, Azmi N, et al. Colorectal peritoneal metastases treated by perioperative systemic chemotherapy and cytoreductive surgery with or without mitomycin-C-based HIPEC. A comparative study using the peritoneal surface disease severity score (PSDSS). Ann Surg Oncol. 2019 (in press).

4. Enomoto LM, Choudry MH, Bartlett DL, et al. Outcomes after adjuvant hyperthermic intraperitoneal chemotherapy for high-risk primary appendiceal neoplasms after complete resection. Ann Surg Oncol. 2019. https://doi.org/10.1245/s10434-019-07634-y.

5. Ali YM, Sweeney J, Shen P, et al. Effect of cytoreductive surgery and hyperthermic intraperitoneal chemotherapy on quality of life in patients with peritoneal mesothelioma. Ann Surg Oncol. 2019. https://doi.org/10.1245/s10434-019-07425-5.

6. Bayat Z, Taylor EL, Bischof DA, et al. Impairments in bowel function, social function and quality of life after cytoreductive surgery and hyperthermic intraperitoneal chemotherapy. Ann Surg Oncol. 2019. https://doi.org/10.1245/s10434-019-07385-w.

7. Gamboa AC, Zaidi MY, Lee RM, et al. Optimal surveillance frequency after CRS/HIPEC for appendiceal and colorectal neoplasms: a multi-institutional analysis of the US HIPEC Collaborative. Ann Surg Oncol. 2019. https://doi.org/10.1245/ s10434-019-07526-1.

8. Solomon D, Bekhor E, Leigh N, et al. surveillance of low-grade appendiceal mucinous neoplasms with peritoneal metastases after cytoreductive surgery and hyperthermic intraperitoneal 
chemotherapy: are 5 years enough? A multisite experience. Ann Surg Oncol. 2019. https://doi.org/10.1245/s10434-019-07678-0.

9. Zaidi MY, Lee RM, Gamboa AC, et al. Preoperative risk score for predicting incomplete cytoreduction: a twelve institution study from the US HIPEC Collaborative. Ann Surg Oncol. 2019. https://doi.org/10.1245/s10434-019-07626-y.

10. Sabesan A, Felder S, Feuerlein S, et al. Preoperative radiographic assessment predicts incomplete cytoreduction in patients with low grade mucinous adenocarcinoma of the appendix. Ann Surg Oncol. 2019. https://doi.org/10.1245/s10434-019-07676-2.

11. Stiles ZE, Murphy AJ, Anghelescu DL, et al. Desmoplastic small round cell tumor: Long-term complications after cytoreduction and hyperthermic intraperitoneal chemotherapy. Ann Surg Oncol. 2019. https://doi.org/10.1245/s10434-019-07339-2.

12. Zambrano-Vera K, Sardi A, Munoz-Zuluaga C, et al. Outcomes in peritoneal carcinomatosis from appendiceal goblet cell carcinoma treated with cytoreductive surgery and hyperthermic intraperitoneal chemotherapy (CRS/HIPEC). Ann Surg Oncol. 2019 (in press).

13. Narayan RR, Harris JW, Chou JF, et al. Prediction of recurrence patterns from hepatic parenchymal disease after resection of colorectal liver metastases. Ann Surg Oncol. 2019 (in press).

14. Miura JT, Dossett LA, Thapa R, et al. Robotic-assisted pelvic lymphadenectomy for metastatic melanoma results in durable oncologic outcomes. Ann Surg Oncol. 2019. https://doi.org/10. 1245/s10434-019-07333-8.

Publisher's Note Springer Nature remains neutral with regard to jurisdictional claims in published maps and institutional affiliations. 